\title{
INCLUSÃO DAS SONDAGENS AIRS/AMSU NO SISTEMA GLOBAL DE ASSIMILAÇÃO/ PREVISÃO DE TEMPO DO CPTEC/INPE: ESTUDO DE IMPACTO
}

\author{
RITA VALÉRIA ANDREOLI, RODRIGO AUGUSTO FERREIRA DE SOUZA, \\ LUIZ FERNANDO SAPUCCI, SÉRGIO HENRIQUE F. SOARES, \\ DIRCEU LUIS HERDIES, JOSÉ ANTONIO ARAVÉQUIA
}

\author{
Instituto Nacional de Pesquisas Espaciais (INPE) \\ Centro de Previsão de Tempo e Estudos Climáticos (CPTEC) \\ Rodovia Presidente Dutra, km 39 SP-RJ, 12630-000, Cachoeira Paulista - SP, Brasil. \\ rita@cptec.inpe.br, rodrigo@cptec.inpe.br, sapucci@cptec.inpe.br \\ sergih@cptec.inpe.br, dirceu@cptec.inpe.br, araveq@cptec.inpe.br
}

Recebido Novembro 2006 - Aceito Maio 2007

\begin{abstract}
RESUMO
Sondagens remotas têm sido consideradas como fontes primárias de informação para fornecer uma boa estimativa das condições iniciais para modelos de Previsão Numérica de Tempo. Neste contexto, o objetivo deste estudo é avaliar o impacto das sondagens AIRS/AMSU no sistema de assimilação/previsão de tempo do Centro de Previsão de Tempo e Estudos Climáticos do Instituto Nacional de Pesquisas Espaciais (CPTEC/INPE). O impacto é quantificado em termos das diferenças entre as previsões e o campo de análise e entre as previsões e as observações. Os resultados indicam claramente uma melhora na qualidade das análises e previsões, principalmente em regiões onde o número de observações convencionais é baixo. Além disso, são notadas melhorias significativas na performance do sistema de assimilação/previsão de tempo sobre o Hemisfério Sul e América do Sul, comparado com os experimentos sem a inclusão dos dados. A melhora na performance desse sistema em 4 dias para o Hemisfério Sul é equivalente a ganhar uma extensão da capacidade de previsão em torno de 6 horas. Estes resultados mostram que esse novo sistema de sondagem tem um potencial significativo para melhorar o desempenho do sistema de assimilação/previsão de tempo do CPTEC e prover, operacionalmente, melhores previsões de tempo.
\end{abstract}

Palavras Chave: Perfis Atmosféricos, Assimilação de dados, Previsão Numérica de Tempo.

ABSTRACT: INCLUSION OF THE AIRS/AMSU SOUNDING IN THE CPTEC GLOBAL DATA ASSIMILATION/FORECAST SYSTEM: IMPACT STUDY.

Remote sensing data have become the predominant source of information for providing good estimation of the initial conditions for Numerical Weather Prediction. The purpose of this study is to evaluate the impact of the AIRS/AMSU sounding on the CPTEC global data assimilation/forecast system. The impact is evaluated in terms of the difference between the forecasts and analysis fields. The results clearly indicate an improvement in the quality of the analysis and forecasts, mainly in the Southern Hemisphere, when AIRS/AMSU soundings are included in the assimilation/forecast system. Besides, the results from the initial experiments with AIRS/AMSU data indicate significant improvements in forecast skill over the Southern Hemisphere and South America, compared with the experiment without AIRS/AMSU sounding data. The improvement in the forecast skills at four days in Southern Hemisphere is equivalent to gaining an extension of forecast capability of six hours. These results indicate the potential of AIRS/AMSU sounding system to improve operational forecast skill at CPTEC/INPE.

Keywords: Atmospheric Profile, data assimilation, weather prediction 


\section{INTRODUÇÃO}

Durante os últimos anos, o ganho na qualidade das condições iniciais do estado da atmosfera, ou análises, utilizadas por modelos de Previsão Numérica de Tempo (PNT) tornouse um aspecto crítico para o aprimoramento das previsões de tempo. Observações convencionais dos parâmetros atmosféricos são, obviamente, o principal ingrediente para uma boa análise. Observações de satélite que foram durante algum tempo o principal contribuinte de informações sobre o Hemisfério Sul, devido a escassez de dados convencionais nessa região, são consideradas atualmente como fonte primária de informações para modelos de PNT para todo o globo (Zapotocny et al., 2005; Kelly et al., 2004).

O uso de dados de satélite em modelos de PNT vem desde 1969, quando observações a partir do primeiro sondador de temperatura, o Space Infrared Sounder (SIRS-A), a bordo do satélite Nimbus 3, foram introduzidas. Pouco depois, em 1973, informações de ventos a partir de observações de deslocamento de nuvens obtidas por satélites geoestacionários foram adicionadas. Desde o início, as sondagens remotas tiveram um impacto positivo na previsão de tempo, principalmente, no Hemisfério Sul, onde as estações de coleta de dados convencionais são esparsas (Ohring, 1979; Eyre, 1993; Joiner e da Silva, 1998; Errico et al., 2000; Atlas, 2005). Por essas razões, esforços constantes têm sido aplicados para incorporar mais observações, especialmente de satélites, nas análises operacionais em diversos Centros de previsão de tempo.

Atualmente, no Centro de Previsão de Tempo e Estudos Climáticos (CPTEC) os dados de satélites compreendem, aproximadamente, $85 \%$ das observações usados para inicializar modelos globais de previsão de tempo. Mesmo assim, isto representa uma pequena fração das informações de satélites disponíveis. Dentre os sensores disponíveis, o sondador atmosférico Atmospheric Infrared Sounder (AIRS), a bordo do satélite AQUA, lançado pela National Aeronautic and Space Administration (NASA) em 2002, é o primeiro instrumento da nova geração de sensores hiperespectrais de radiação infravermelha, cuja finalidade principal é fornecer informações de boa qualidade para inferência de perfis atmosféricos de temperatura e umidade e dele espera-se prover melhoramentos substanciais, especialmente nas medidas de temperatura, em relação aos instrumentos anteriores, tal que quando assimiladas em modelos de circulação geral, melhoramentos significativos na performance das previsões possam ser alcançados (Parkinson, 2003; Aumann et al., 2003). Em relação à qualidade dos perfis de temperatura inferidos pelo Advanced TIROS-N/NOAA Operational Vertical Sounder (ATOVS), as informações inferidas a partir do sensor AIRS em conjunto com o sensor Advanced Microwave Sounding Unit (AMSU), que juntos compõem o sistema de sondagem AIRS/AMSU, mostram uma melhora de no mínimo $0,5 \mathrm{~K}$ (para uma camada vertical de 1 $\mathrm{km}$ ) quando comparados com os dados de radiossondagens (Divakarla et al., 2006). Além disso, os perfis atmosféricos inferidos pelo sistema de sondagem AIRS/AMSU têm sido validados sobre superfícies oceânicas e continentais com um erro médio quadrático para o perfil de temperatura, variando de $0,6 \mathrm{~K}$ a $1 \mathrm{~K}$, em regiões oceânicas entre $50^{\circ} \mathrm{Ne} 50^{\circ} \mathrm{S}$, e de $0,9 \mathrm{~K}$ a 1,3 K para a região global (Fetzer et al., 2005). Particularmente sobre a América do Sul, o erro médio quadrático para o perfil de temperatura atinge valores inferiores a $2 \mathrm{~K}$, exceto próximo à superfície onde há uma ligeira degradação (Souza et al., 2006). No entanto, as sondagens AIRS/AMSU ainda não estão incluídas operacionalmente no processo de assimilação de dados do CPTEC. Desde que uma das limitações da previsão de tempo é a escassez de observações sobre algumas regiões oceânicas e continentais, principalmente no Hemisfério Sul (HS), uma alternativa para esse problema é a utilização das sondagens remotas obtidas a partir do satélite AQUA. Desta forma, estas podem atuar para minimizar o problema de escassez de dados, em regiões onde, tradicionalmente, não existem informações de radiossondas.

Nesse contexto, este estudo apresenta os resultados preliminares relacionados à inclusão dos perfis atmosféricos, obtidos a partir de informações do sistema de sondagem AIRS/AMSU, no sistema de assimilação/previsão do tempo do CPTEC/INPE, principalmente no que concerne a análise de impacto. A próxima seção descreve os experimentos realizados, assim como a metodologia de avaliação. Na seção 3 , são apresentados os resultados relacionados à performance do modelo, bem como os relacionados à análise de impacto. As conclusões são apresentadas na seção 4.

\section{EXPERIMENTOS NUMÉRICOS E METODOLOGIA DE AVALIAÇÃO}

Neste estudo foram realizados experimentos de assimilação/previsão de tempo no CPTEC/INPE utilizando o Global Physical-space Statistical Analysis System (GPSAS, Herdies et al., 2002), que representa uma combinação do Modelo Atmosférico de Circulação Geral do CPTEC (MGCA-CPTEC/ COLA) e o esquema de assimilação de dados Physical-space Statistical Analysis System (PSAS, Da Silva et al., 1995). Os resultados apresentados a seguir foram gerados com a versão de 2003 do PSAS e o modelo global de previsão CPTEC/COLA, com uma resolução T126 L28, que corresponde à resolução horizontal de $100 \mathrm{~km}$ no equador, com 28 níveis em coordenada sigma na vertical. Essa nova versão do sistema de assimilação/ previsão está em fase de operacionalização. Detalhes do MCGA-CPTEC/COLA e do sistema de assimilação de dados 
usados neste estudo podem ser encontrados nos trabalhos de Cavalcanti et al. (2002) e Cohn et al. (1998), respectivamente.

Dois experimentos de previsão são realizados com o objetivo de avaliar o impacto da inclusão das sondagens AIRS/AMSU no sistema de assimilação/previsão. Em um primeiro experimento, denominado C_AIRS (com a inclusão das sondagens AIRS/AMSU), dados convencionais tais como, altura geopotencial, pressão em superfície, umidade específica e vento zonal e meridional oriundos do Global Telecommunication System (GTS), água precipitável obtidas a partir do Special Sensor Microwave Imager (SSM/I), dados de vento sobre o oceano (Nasa's Quik Scatterometer), informações de temperatura e umidade do Advanced TIROS-N/NOAA Operational Vertical Sounder (ATOVS) e perfis de altura geopotencial (sondagens AIRS/AMSU) obtidos a partir do algoritmo de inversão da NASA (Susskind, et al. 2003) são assimilados pelo sistema. No segundo experimento denominado S_AIRS (sem a inclusão das sondagens AIRS/AMSU), as informações do sistema de sondagem AIRS/AMSU sobre o globo são excluídas do processo de assimilação. Esses experimentos foram realizados em um modo cíclico, no qual, inicialmente, a partir de uma previsão de 6 horas (first-guess) o sistema de análise PSAS gera uma nova estimativa do estado da atmosfera num dado instante de tempo. Essa nova estimativa, ou condição inicial servirá de base para a nova rodada do modelo e a partir de então se inicia o ciclo de previsões (Herdies et al., 2002). Previsões para até cinco (5) dias foram realizadas para o período 01/03/2004 a 31/03/2004.

Tipicamente, uma órbita do satélite AQUA cobre diferentes regiões do globo. A Figura 1 mostra a distribuição espacial dos perfis AIRS/AMSU, efetivamente assimilados, nos horários das 06 e 12 UTC, em 15 de Março de 2004. Como as passagens das 18 UTC cobrem basicamente as mesmas regiões correspondentes às passagens das 06 UTC (o mesmo ocorrendo para as passagens das 00 e 12 UTC) optou-se em realizar a avaliação do impacto, para dois subconjuntos de análises e previsões, sendo um subconjunto dos horários das 06 e 18 UTC e o outro dos horários das 00 e 12 UTC.Inicialmente, a sensibilidade do GPSAS à inclusão dos perfis AIRS/AMSU é calculada separadamente para os campos de análises dos subconjuntos definidos anteriormente. Essa sensibilidade no erro médio absoluto é definida como:

$$
G=\frac{\sum_{i=1}^{N}\left|A 1_{i}-A 2_{i}\right|}{N}
$$

onde $\mathrm{N}$ é o número de rodadas de previsão do modelo (30 rodadas para cada horário do campo de análise, totalizando 60 rodadas para cada subconjunto). As variáveis A1 e A2 são as análises dos experimentos S_AIRS e C_AIRS, respectivamente. Desde que este cálculo considera apenas as análises dos experimentos 1 e 2, o mesmo não indica se os campos de análises são melhores ou piores com a inclusão do dados AIRS/AMSU, mas sim a distribuição espacial do erro médio absoluto entre os experimentos realizados.

Para se ter uma idéia da qualidade desses campos de análises, os mesmos são comparados com as análises do National Centers for Environmental Prediction (NCEP). Neste caso, somente serão apresentados os resultados para os campos médios para os horários de 00 e 12 UTC. As análises do NCEP são utilizadas para verificação pelo fato dessas serem utilizadas,
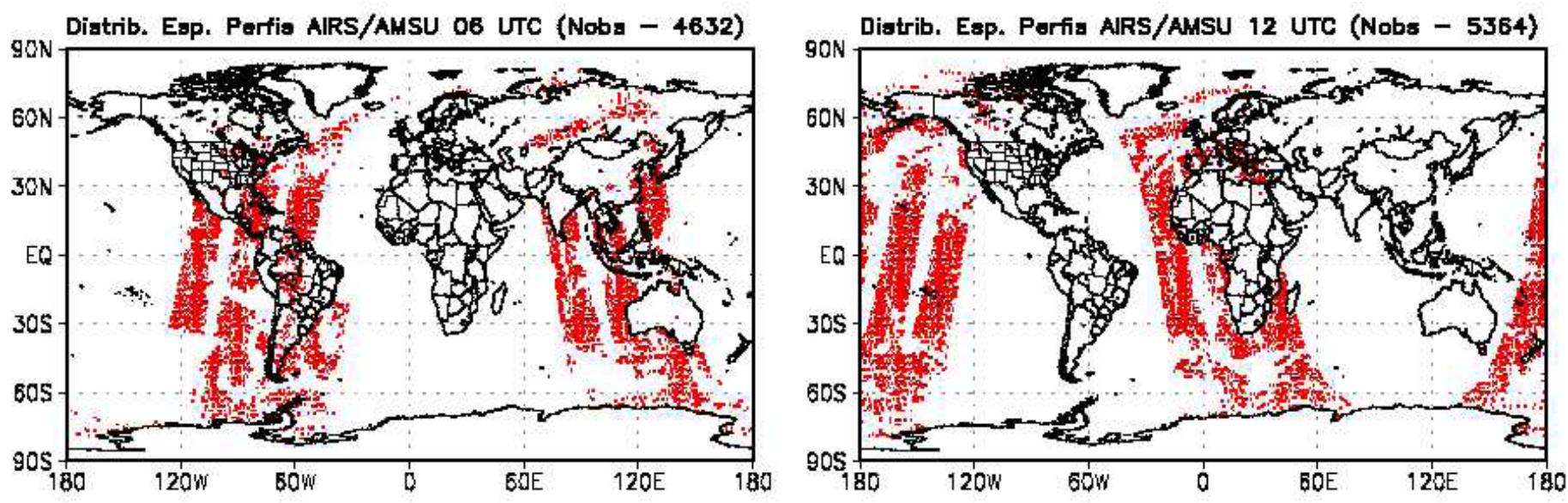

Figura 1 -Distribuição espacial dos perfis AIRS/AMSU assimilados nos horários das 06 UTC (à esquerda) e 12 UTC (à direita), em 15 de Março de 2004. 
paralelamente, para inicialização operacional do Modelo Global do CPTEC. Nessa avaliação utiliza-se uma estatística baseada no erro médio quadrático (descrita abaixo), com o diferencial que os campos espaciais foram gerados substituindo a média espacial (equação 2) pela média sobre todas as rodadas do modelo (60 rodadas que juntas correspondem às análises dos horários das 00 e 12 UTC) e as análises de referência utilizadas referem-se às do NCEP.

A performance do modelo e a qualidade das previsões são apresentadas em função do coeficiente de Correlação de Anomalias (CA) e do índice de impacto, considerando somente as previsões obtidas a partir das condições iniciais das $00 \mathrm{e}$ 12 UTC. Usualmente, quando se avalia a inclusão de novas observações no sistema de assimilação/previsão de tempo, cada experimento (com ou sem a inclusão de novas observações) é avaliado de encontro às suas próprias análises. Contudo, no contexto de estudos de impacto, uma representação mais precisa de cada tipo de observação é obtida quando a estimativa do estado da atmosfera, que melhor represente o campo observado, é usado para a avaliação. Neste estudo utiliza-se o campo de análise do experimento C_AIRS como referência, tanto para os cálculos da CA quanto para os índices de impacto. Índices de impacto das previsões em relação aos dados de radiossondas sobre a região da América do Sul (AS) são também apresentados. Neste caso, os campos do modelo foram interpolados para o mesmo ponto de observação e as diferenças entre as previsões e as observações foram utilizadas.

Como em Zapotocny et al. (2005), os índices de impactos (IP) positivos/negativos na previsão são determinados pela equação:

$$
\left.I P=100 \times\left\{\sqrt{\frac{\sum_{i=1}^{N}\left(D_{i}-A 2_{i}\right)^{2}}{N}}-\sqrt{\frac{\sum_{i=1}^{N}\left(C_{i}-A 2_{i}\right)^{2}}{N}}\right] \div \sqrt{\frac{\sum_{i=1}^{N}\left(C_{i}-A 2_{i}\right)}{N}}\right\}
$$

onde $\mathrm{N}$ é o número total de pontos de grade na superfície isobárica da análise de referência (total de 3630 pontos para a região da $\mathrm{AS}$, entre $10^{\circ} \mathrm{N}-50^{\circ} \mathrm{S}$ e $\left.80^{\circ} \mathrm{W}-30^{\circ} \mathrm{W}\right)$. Para avaliação em relação às radiossondagens, $\mathrm{N}$ representa o número de informações disponíveis. As variáveis $\mathrm{C}$ e $\mathrm{D}$ são as previsões dos experimentos C_AIRS e S_AIRS, respectivamente, e A é o campo de análise ou a radiossondagem usada para verificação. Um índice de impacto positivo significa uma melhora na previsão com a inclusão das sondagens AIRS/AMSU. A média temporal do IP exclui os primeiros 5 (cinco) dias de cada experimento. Esta redução é feita com intuito de eliminar o impacto das sondagens AIRS/AMSU nas primeiras rodadas do modelo e reduz o período de análise para 26 dias. Adicionalmente, campos espaciais foram gerados substituindo a média espacial (equação 2) pela média sobre todas as rodadas de previsão do modelo (51 rodadas que juntas correspondem às previsões geradas a partir das condições iniciais dos horários das 00 e 12 UTC).

\section{RESULTADOS}

\subsection{Avaliação dos Campos de Análises}

A Figura 2 mostra a distribuição espacial da média mensal do erro médio absoluto da temperatura, entre as análises dos experimentos $\mathrm{C}$ _AIRS e S_AIRS. As Figuras 2a, 2b e 2 c se referem nos níveis de $850 \mathrm{hPa}, 500 \mathrm{hPa}$ e $300 \mathrm{hPa}$, respectivamente, para os horários das 06 e 18 UTC e de forma similar as Figuras 2d, 2e e $2 \mathrm{f}$ se referem aos horários das 00 e 12 UTC. Em geral, essas figuras indicam que as regiões mais favoráveis às variações nos campos de análises do GPSAS, coincidem com aquelas da órbita do satélite AQUA, ou seja, regiões onde efetivamente estão sendo incluídas as informações oriundas desse satélite.

Para os campos médios dos horários das 06 e 18 UTC, a Figura 2a mostra que os maiores erros na temperatura em $850 \mathrm{hPa}$ ocorrem nas regiões polares com erros superiores a $3 \mathrm{~K}$, em regiões do Pacífico subtropical leste, do Índico entre $30^{\circ} \mathrm{S}-60^{\circ} \mathrm{S}$, e no sul da Índia com erros da ordem de 0,9 a 1,5 $\mathrm{K}$. Menores erros, da ordem de 0,6 K, abrangem extensas regiões do globo. Na comparação entre as Figuras 2a, 2b e 2c observa-se que as maiores diferenças no campo de temperatura, devido à inclusão das sondagens AIRS/AMSU, ocorrem na baixa troposfera $(850 \mathrm{hPa})$ sobre regiões oceânicas do HS, enquanto sobre regiões da América do Sul, os maiores erros no campo de temperatura ocorrem no nível de $500 \mathrm{hPa}$ e atingem valores máximos no intervalo de 0,9 a 1,5 K. Máximos erros nas regiões oceânicas do HS, e particularmente sobre a América do Sul refletem a escassez de informações convencionais nessas regiões. A distribuição espacial dos erros médios absolutos para temperatura em $850 \mathrm{hPa}$, média para os horários das 00 e 12 UTC (Figura 2d), mostra maiores valores em regiões oceânicas globais e continentais do HS, onde existe uma falta de dados convencionais. Por outro lado, em regiões continentais do Hemisfério Norte (HN), são encontrados menores erros (abaixo de $0,3 \mathrm{~K}$ ), pelo fato de existir nessas regiões uma maior concentração de dados de radiossondagens. Semelhante ao que ocorre para os campos médios dos horários das 06 e 18 UTC, o erro médio absoluto no campo de temperatura diminui com a altura (Figuras 2e e 2f), quando as sondagens AIRS/AMSU são incluídas no sistema de assimilação de dados. Nota-se ainda, nas Figuras 2d, 2e e 2f, uma sensibilidade nos campos de temperatura em relação à inclusão dos perfis AIRS/AMSU, em regiões onde, efetivamente, esses perfis não são incluídos, como é o caso da região da América do Sul. Isso ocorre pelo fato dos experimentos serem executados em um modo cíclico. 

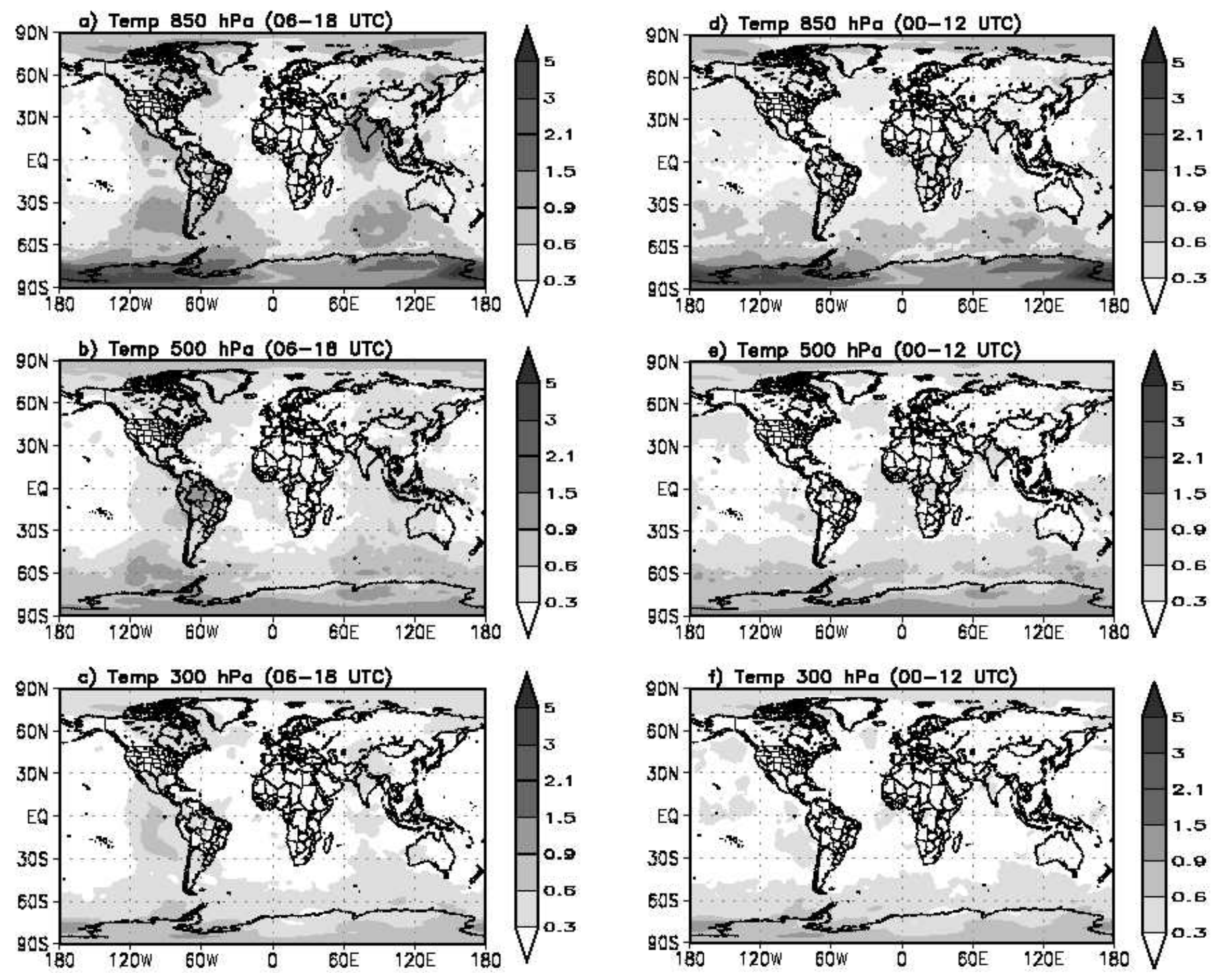

Figura 2 - Distribuição espacial da média mensal do erro absoluto da temperatura (K), médio dos horários das 06 e 18 UTC, para os níveis de (a) $850 \mathrm{hPa}$, (b) $500 \mathrm{hPa}$ e (c) $300 \mathrm{hPa}$; e médio dos horários das 00 e 12 UTC para os níveis de: (d) $850 \mathrm{hPa}$, (e) $500 \mathrm{hPa}$ e (f) $300 \mathrm{hPa}$. Esses erros são calculados para o período de 02/03/2004 a 31/03/2004.

Considerando como referência as análises do NCEP (horários das 00 e 12 UTC) a Figura 3 ilustra o impacto (\%) gerado pela inclusão das sondagens AIRS/AMSU nos campos de temperatura obtidos pelo GPSAS. Em $850 \mathrm{hPa}$, é notada uma melhora nos campos de análise de temperatura (superior a $15 \%$ ) sobre a América do Sul e grandes extensões oceânicas do HS. Impacto negativo é notado na região do Atlântico Equatorial, próximo a costa da África. Nas demais regiões o impacto é menor (inferior a 15\%) (Figura 3a). Para o nível de $500 \mathrm{hPa}$ (Figura 3b), impactos positivos sobre a América do Sul, na parte sul da África e no Atlântico Norte atingem valores médios em torno de 5 a $15 \%$ (contornos não mostrados na figura). Além disso, impactos positivos maiores que $15 \%$ são notados em regiões oceânicas ao sul de $30^{\circ} \mathrm{S}$. Diferente do que ocorre na baixa e média troposfera, no nível de 300 $\mathrm{hPa}$ impactos positivos, maiores que $15 \%$ restringem-se à região do Pacífico tropical leste e espalhados em pequenas regiões oceânicas sobre o globo (Figura 3c). Particularmente sobre a América do Sul, impactos positivos na faixa de 5 a $15 \%$ (contornos não mostrados na figura) são notados na parte sul (regiões centro oeste e sul do Brasil, Uruguai, Paraguai e Argentina). As avaliações para os horários das 06 e 18 UTC (figuras não mostradas) mostram que os maiores impactos ocorrem nas regiões que apresentam os maiores valores de erro médio absoluto (Figuras 2 a-c), e chegam a atingir um fator de impacto 2 vezes maior do que àqueles encontrados nas 
análises das 00 e 12 UTC. Esses resultados sugerem que, para os campos de temperatura, a condição inicial ou análises obtida com o experimento C_AIRS fisicamente apresenta uma maior concordância com a análise do NCEP.


Figura 3 - Distribuição espacial do índice de impacto (\%) no campo de análise da temperatura devido a inclusão dos perfis AIRS/AMSU, em relação às análises do NCEP, média para os horários das 00 e 12 UTC no período de 02/03/2004 a 31/06/2004, para os níveis de: (a) $850 \mathrm{hPa}$, (b) $500 \mathrm{hPa}$, (c) $300 \mathrm{hPa}$. Os contornos contínuos (tracejados) usados são 15,35 e $65(-15,-35,-65)$. Sombreados claros (escuros) correspondem a valores inferiores (superiores) a -15 (15).
Alterações no campo de umidade específica relativas à inclusão das sondagens AIRS/AMSU são apresentadas na Figura 4, somente para o nível de $850 \mathrm{hPa}$, onde é maior a concentração de umidade. Nesta figura, os máximos valores de erro médio absoluto ocorrem sobre regiões continentais da América do Sul e África e ao longo da Zona de Convergência Intertropical (ZCIT) no Atlântico Tropical, tanto para as análises das 00 e 12 UTC (Figura 4), quanto para as das 06 e 18 UTC (figura não mostrada). Em relação às análises do NCEP (Figura 5) essa sensibilidade no campo de umidade específica reflete um impacto positivo na qualidade das análises nas regiões continentais, enquanto que para a região do Atlântico equatorial leste o impacto é negativo (aproximadamente -5 a -15\%). Padrões similares são encontrados para as análises das 06 e 18 UTC (figuras não mostradas).

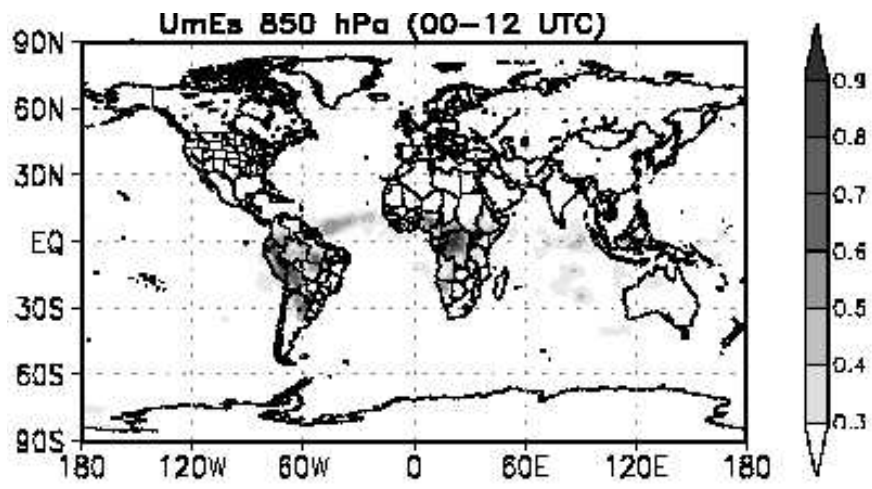

Figura 4 - Distribuição espacial da média mensal do erro absoluto da umidade específica $(\mathrm{g} / \mathrm{kg})$, médio dos horários das 00 e 12 UTC, no período de 02/03/2004 a 31/03/2004 para nível de $850 \mathrm{hPa}$.

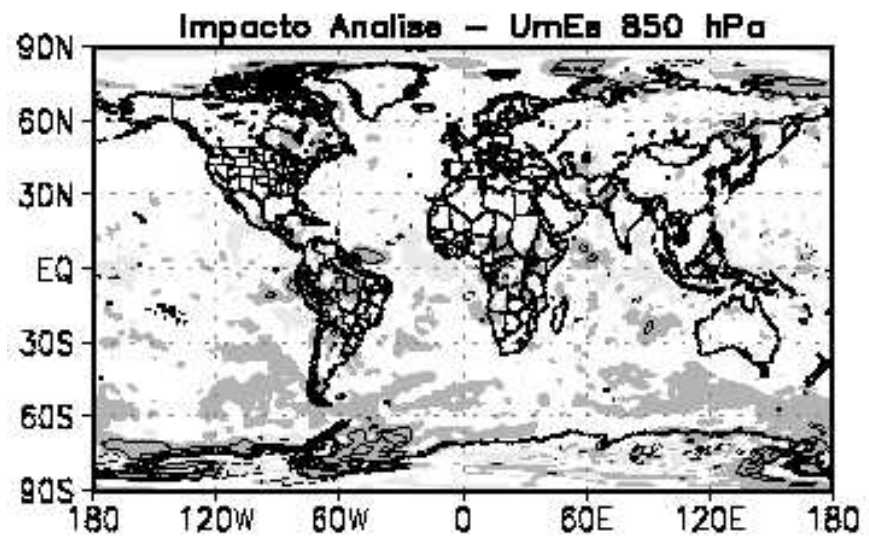

Figura 5 - Distribuição espacial do índice de impacto (\%) no campo de análise da umidade específica, devido a inclusão dos perfis AIRS/ AMSU, em relação às análises do NCEP, média para os horários das 00 e 12 UTC, para o período de 02/03/2004 a 31/06/2004. Os contornos são os mesmos da Figura 3 , exceto que os sombreados claros (escuros) correspondem a valores inferiores (superiores) a -5 (5). 
Similar aos resultados encontrados para temperatura, as maiores diferenças médias mensais na componente zonal do vento média dos horários das 06 e 18 UTC e das 00 e 12 UTC em $850 \mathrm{hPa}$ (Figura 6a e 6d, respectivamente) ocorrem em regiões oceânicas do HS e sobre regiões da América do Sul e África. Sobre as regiões da América do Norte, Europa e Austrália as diferenças no campo de vento zonal são pequenas (inferior a 0,5 $\mathrm{m} / \mathrm{s}$ ). Novamente, isso pode ser devido a maior concentração de dados convencionais sobre essas regiões. Nos níveis de $500 \mathrm{hPa}$ e $300 \mathrm{hPa}$ (Figuras 6b, 6c, 6e e 6f ), os erros médios absolutos acima de $0,5 \mathrm{~m} / \mathrm{s}$ aparecem em quase todo o globo,
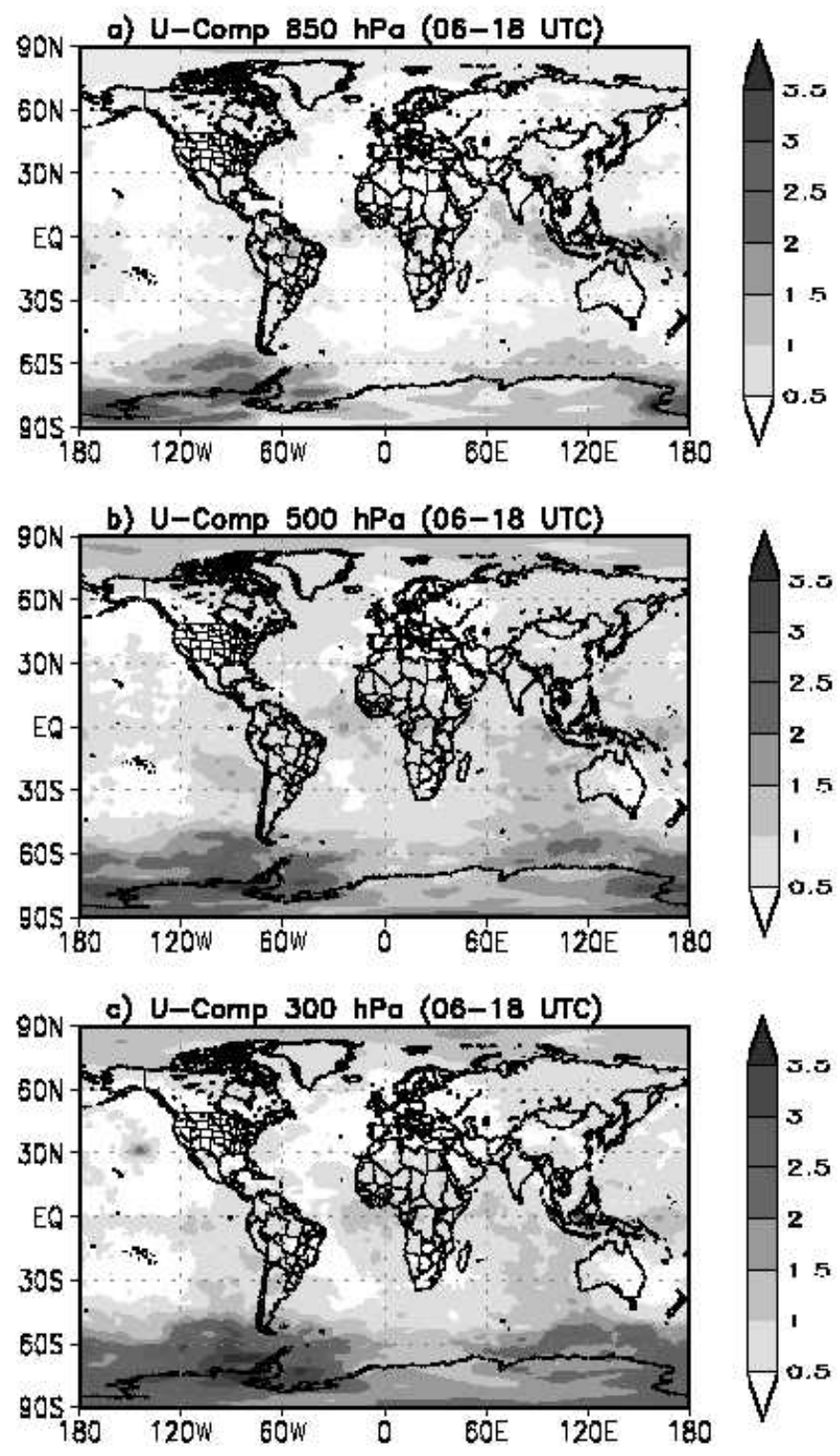

Figura 6 - Idem Figura 2, exceto para a componente zonal do vento. exceto na região da América do Norte, Europa e Austrália, onde as diferenças nos campos de análise são inferiores as esse valor. Comparando com as análises do NCEP para os horários das 00 e 12 UTC, os resultados obtidos com o experimento C_AIRS definem melhor o vento zonal, com um ganho na qualidade dos mesmos em torno de 10 a $15 \%$ em diversas regiões do globo, principalmente, ao sul de $30^{\circ} \mathrm{S}$ para o nível de $850 \mathrm{hPa}$ (Figura 7a), e acima de 15\% para o nível de 300 hPa (Figura 7c). Padrões similares são encontrados para os campos das 06 e 18 UTC (figuras não mostradas).


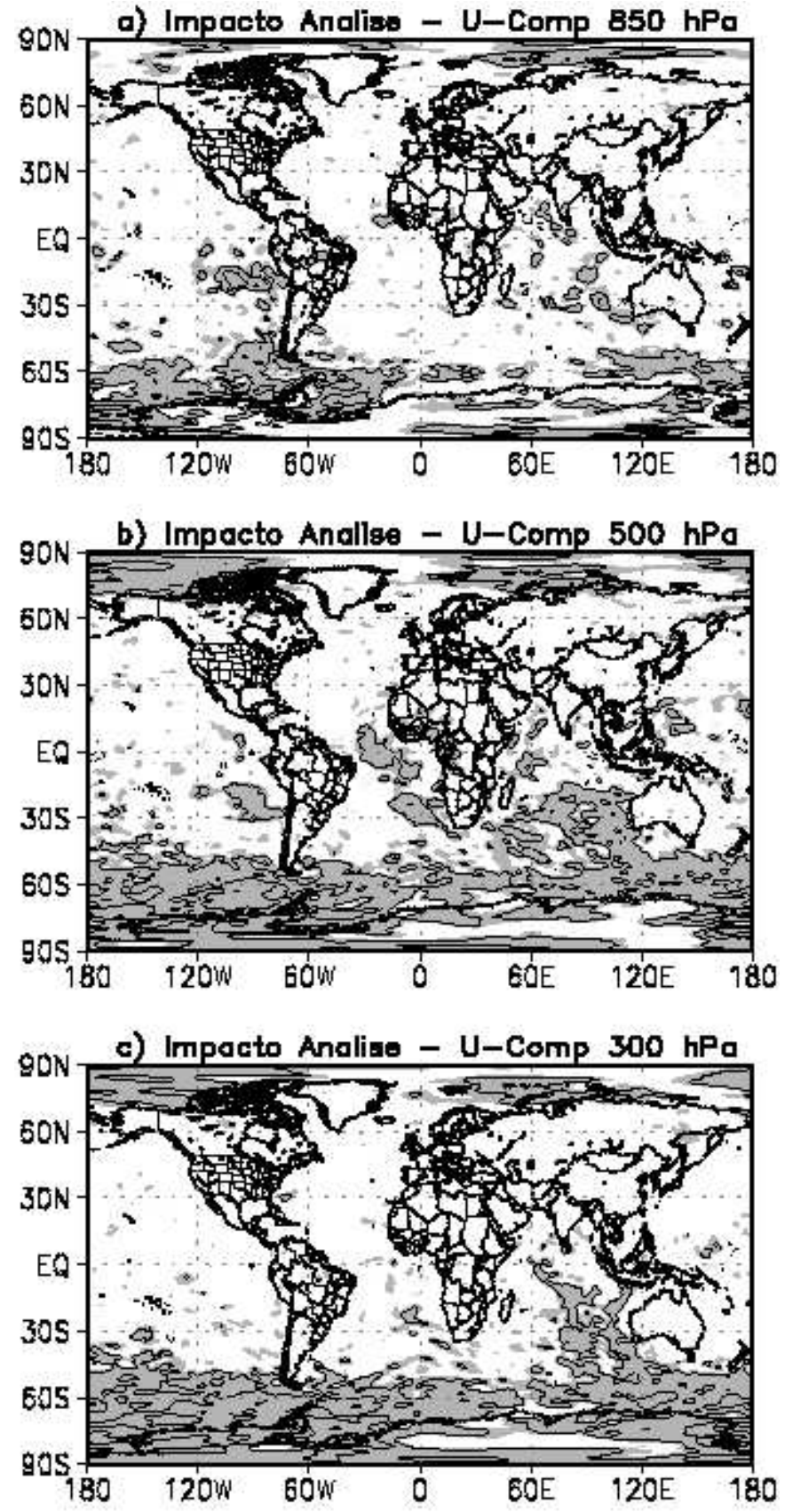

Figura 7 - Idem Figura 3, exceto para a componente zonal do vento. Sombreados claros (escuros) correspondem a valores inferiores (superiores) a -10 (10).

\subsection{IMPACTO NAS PREVISÕES}

Diferente dos cálculos anteriores que avaliam somente a sensibilidade das condições iniciais do GPSAS em relação a inclusão das sondagens AIRS/AMSU, esta seção examina qual é o impacto desses dados na previsão de tempo. As Figuras $8 \mathrm{a}$ e $8 \mathrm{~b}(8 \mathrm{c}$ e $8 \mathrm{~d})$ mostram os coeficientes de CA da altura geopotencial em $850 \mathrm{hPa}(500 \mathrm{hPa})$ para o MCGA- CPTEC/ COLA sobre a região da América do Sul e do HS, entre $20^{\circ} \mathrm{S}$ - $80^{\circ} \mathrm{S}$, respectivamente, para previsões de 1 a 5 dias com e sem a inclusão das sondagem AIRS/AMSU. As correlações de anomalias representam uma média temporal dos impactos positivos/negativos. A inclusão das sondagens AIRS/AMSU, em média, tem um impacto positivo na performance das previsões sobre o HS a partir de 12 horas de previsão durante este período, com ganho de 6 horas de extensão da capacidade de previsão de 4 (quatro) dias. Restringindo-se à região da América do Sul, impactos positivos surgem a partir de 24 horas de previsão e o ganho da extensão da capacidade de previsão para previsões de 5 (cinco) dias é de aproximadamente 12 horas. Análises para a região do Hemisfério Norte (HN) não apresentam, em média, um impacto significativo na performance das previsões quando esses dados são incluídos no processo de assimilação (figuras não mostradas). Resultados similares, para as regiões dos HN e HS, foram mostrados por Atlas (2005), que avaliou o impacto das sondagens AIRS/AMSU utilizando o sistema de assimilação de dados FVSSI, o qual representa uma combinação do modelo de circulação geral da NASA com o sistema de assimilação de dados (Spectral Statistical Interpolation, SSI) da NOAA/NCEP.

Em adição aos cálculos de correlação de anomalias, a Figura 9 mostra o índice de impacto (\%) nas previsões para a altura geopotencial em 850,500 e $250 \mathrm{hPa}$, para a componente zonal do vento em 850 e $250 \mathrm{hPa}$, e para a umidade específica em $850 \mathrm{hPa}$, obtidos em relação ao campo observado (radiossondas), somente considerando a condição inicial das 12 UTC. Para todas as variáveis analisadas, há um impacto positivo da assimilação dos perfis AIRS/AMSU, sobre a região da América do Sul, indicando que as análises/previsões obtidas com o experimento C AIRS, fisicamente, apresentam uma maior concordância com os dados observados. Isto justifica a escolha de se utilizar os campos de análises do experimento C_AIRS como referência nas avaliações de impacto mostradas a seguir.

Na Figura 10 são ilustradas as médias temporais dos índices de Impactos na Previsão (IP, equação2) para a região da América do Sul, para as previsões de 24, 48, 72 e 96 horas. Nessa figura são consideradas as previsões para a componente zonal do vento, a altura geopotencial, a temperatura e a umidade relativa, nos níveis de 1000, 850, 500, 300 e $200 \mathrm{hPa}$, obtidas a partir das condições iniciais das 00 e 12 UTC. Observa-se que para todas as variáveis analisadas, a inclusão das sondagens AIRS/AMSU teve um impacto positivo nas primeiras 96 horas de previsão, exceto para a temperatura em $300 \mathrm{hPa}$, a componente zonal do vento em baixos níveis e umidade relativa, que apresentaram impactos negativos a partir de 96 horas de previsão. Para a componente zonal do vento, nas primeiras 72 horas o maior impacto ocorre no nível de $500 \mathrm{hPa}$. Para este 

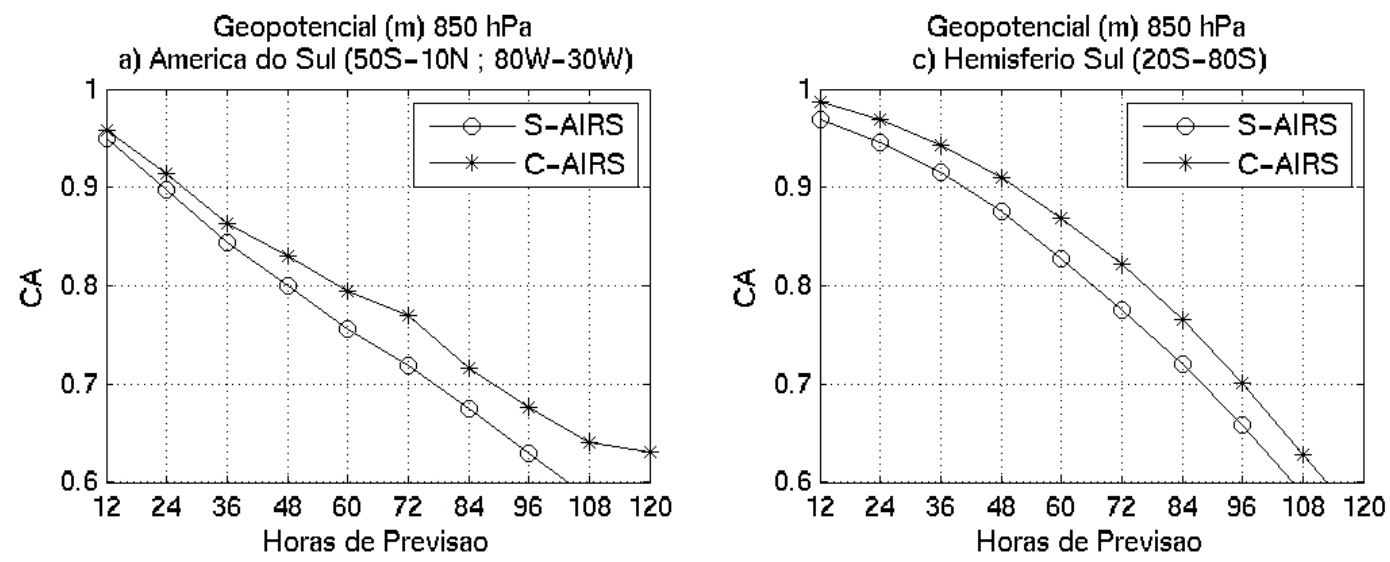

Geopotencial (m) $500 \mathrm{hPa}$ b) America do Sul (50S-10N ; $80 \mathrm{~W}-30 \mathrm{~W})$
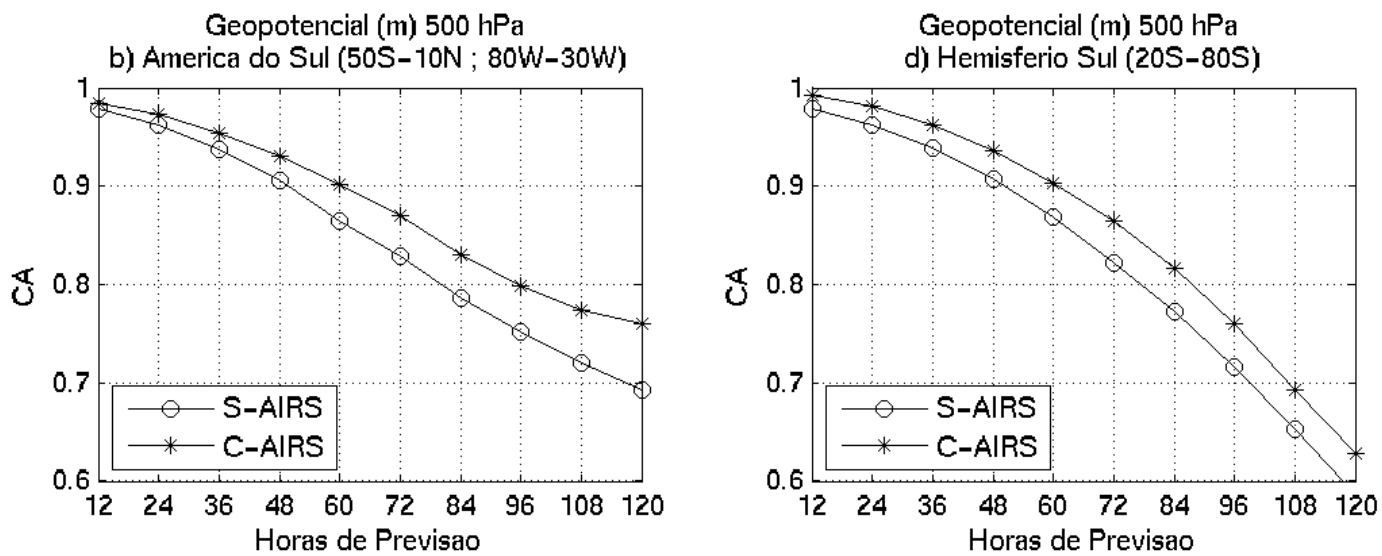

Figura 8 - Coeficiente de Correlação de Anomalias (CA) do geopotencial para MCGA-CPTEC/COLA com e sem a inclusão das sondagens AIRS/ AMSU para as regiões da América do Sul em (a) $850 \mathrm{hPa}$ e (b) $500 \mathrm{hPa}$ e para o Hemisfério Sul em (c) $850 \mathrm{hPa}$ e (d) $500 \mathrm{hPa}$.

nível ainda é notado um ligeiro decréscimo no IP em função do tempo de previsão. Para a umidade relativa o decréscimo no IP em função do tempo de previsão é notado para todos os níveis analisados. Os maiores IP são encontrados para o geopotencial em $500 \mathrm{hPa}$, onde os valores são próximos de $15 \%$ para as previsões de até 72 horas. Outra característica para essa variável é o aumento do impacto na previsão em $200 \mathrm{hPa}$ e $300 \mathrm{hPa}$ em função do tempo de previsão, nas primeiras 72 horas. Para a temperatura, uma característica notável é o aumento do IP em função do tempo de previsão no nível de $850 \mathrm{hPa}$.As Figuras 11 e 12 e 13 mostram a distribuição espacial média mensal do índice de impacto (\%) na previsão sobre a América do Sul para a umidade relativa, a temperatura, a altura geopotencial e a componente zonal do vento, nos níveis de 850,500 e 300 $\mathrm{hPa}$, para 24, 48 e 72 horas de previsão, respectivamente. Para 24 horas de previsão (Figura 11) o maior impacto nas variáveis analisadas ocorre no nível de $500 \mathrm{hPa}$, com valores positivos cobrindo todo o domínio. Restringindo-se às áreas continentais, os maiores impactos (15 a 30\%) para umidade relativa e temperatura em $850 \mathrm{hPa}$ e $300 \mathrm{hPa}$ ocorrem, particularmente, na parte noroeste da América do Sul onde a disponibilidade de observações é baixa. Em $500 \mathrm{hPa}$, impactos positivos na umidade relativa da ordem de 5 a $15 \%$ abrangem toda a região continental, enquanto que para a temperatura impactos positivos restringem-se a região norte da América do Sul.

Para a altura geopotencial, impactos positivos da ordem de 5 a $15 \%$ abrangem toda a região norte e nordeste da América do Sul, para os níveis de 850 e $500 \mathrm{hPa}$. Ao sul de $30^{\circ} \mathrm{S}$, o impacto positivo nas previsões para essa variável pode atingir valores máximos de até $35 \%$, o que indica claramente que a inclusão dos perfis AIRS/AMSU melhora a qualidade das previsões, reduzindo a incerteza em regiões com poucas observações convencionais. Em $300 \mathrm{hPa}$, o padrão espacial do impacto para a altura geopotencial apresenta valores pequenos e regiões com valores de impacto negativo. Para componente zonal do vento, melhorias na previsão atingem valores em torno de 5 a $15 \%$ em diversas regiões da América do Sul nos três níveis analisados. 



Figura 9 - Média temporal dos índice de impacto (\%), sobre a América do Sul, utilizando como referência dados de radiossondagens no horário das 12 UTC.
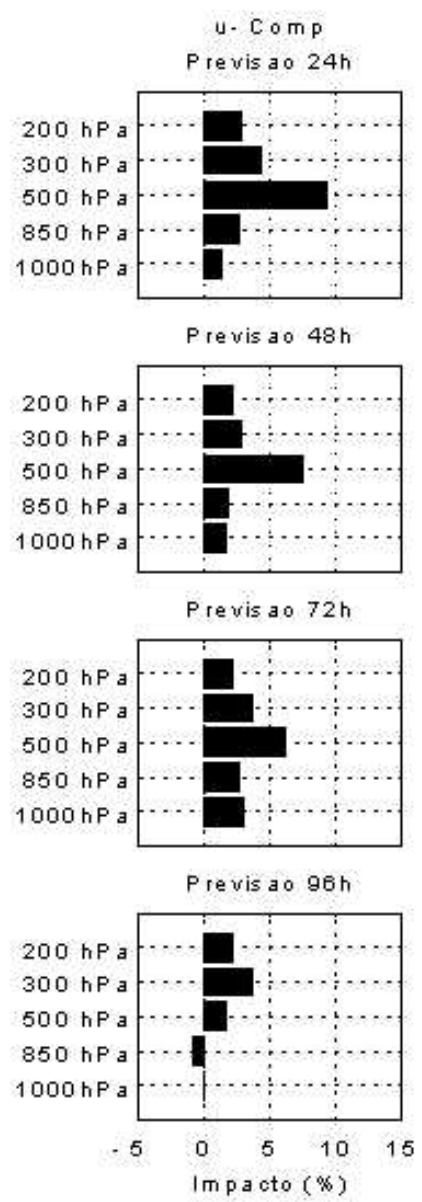

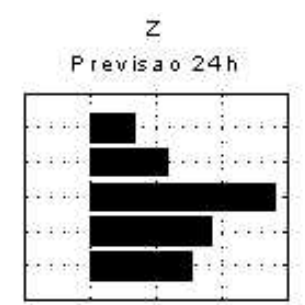

Previsao $48 \mathrm{~h}$

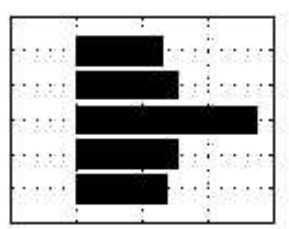

Previsa $072 \mathrm{~h}$

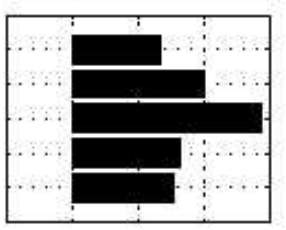

Previsa $96 \mathrm{~h}$

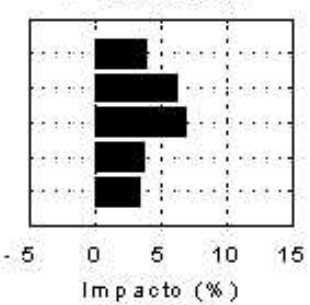

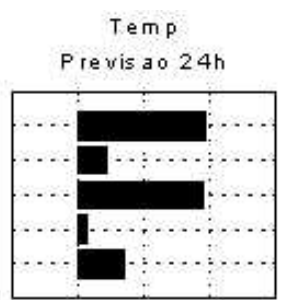

Previs a $48 \mathrm{~h}$

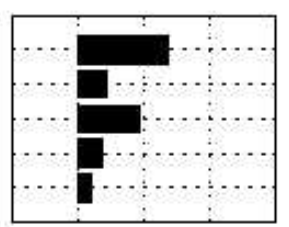

Previs a $72 \mathrm{~h}$

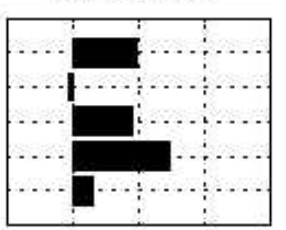

Previs a $0.96 \mathrm{~h}$

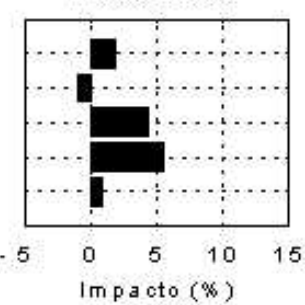

Previs a $024 \mathrm{~h}$

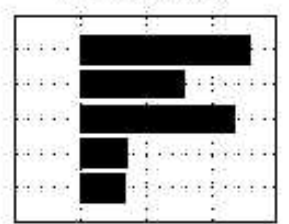

Previs a $48 \mathrm{~h}$

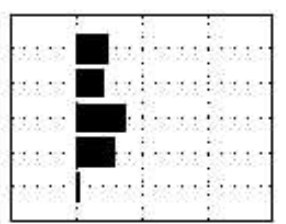

Previsa $072 \mathrm{~h}$

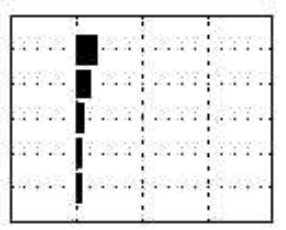

Previs a $096 \mathrm{~h}$

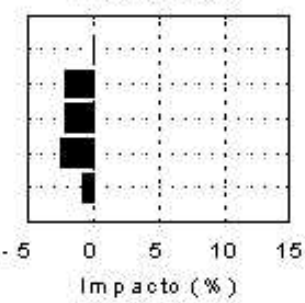

Figura 10 - Média temporal do índice de impacto (\%) nas previsões da 00 e 12 UTC, sobre a América do Sul, para a componente zonal do vento, a altura geopotencial, a temperatura, e a umidade relativa nos níveis de 1000, 850, 500, 300, 200 hPa. Períodos de previsão são $24,48,72$ e 96 horas. 


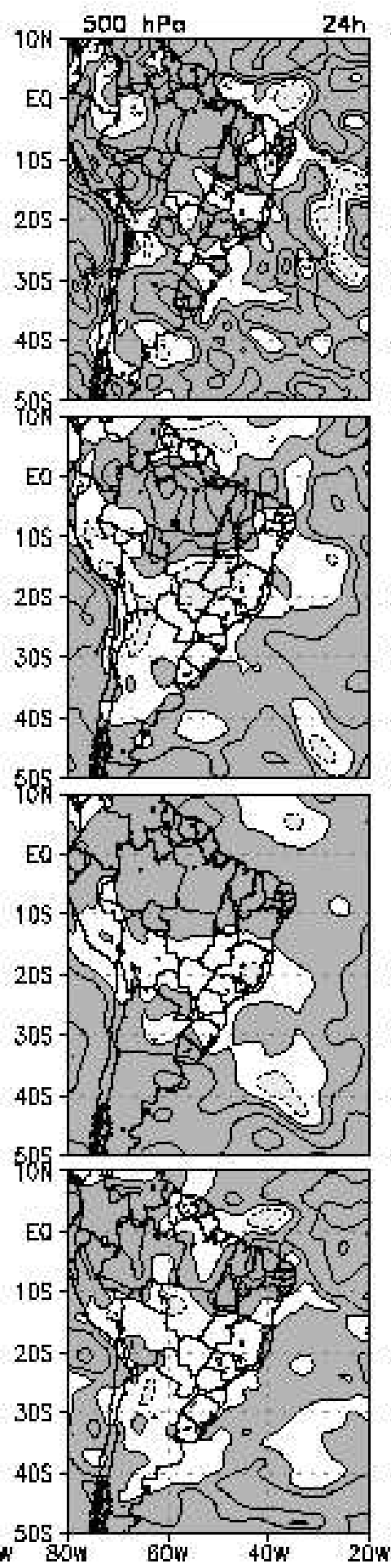

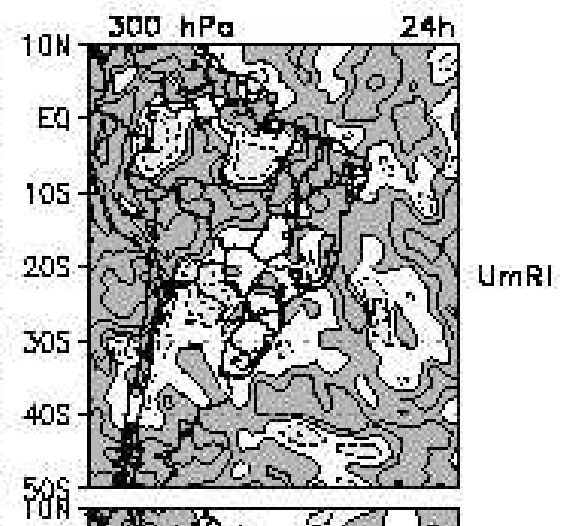

徐



䲞



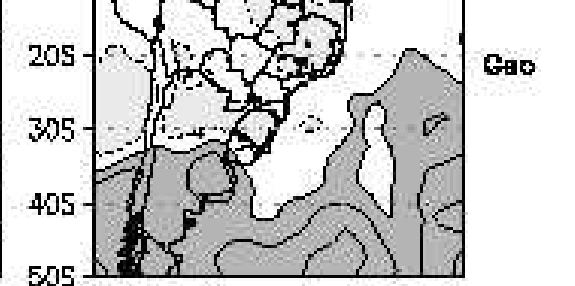

铅

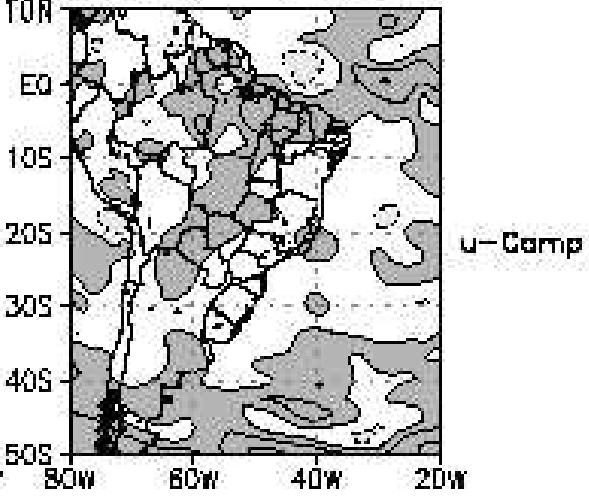

Figura 11 - Distribuição espacial do índice de impacto (\%) na previsão da umidade relativa, da temperatura, da altura geopotencial e da componente zonal do vento para os níveis de $850 \mathrm{hPa}, 500 \mathrm{hPa}$ e $300 \mathrm{hPa}$ para 24 horas de integração. Contornos contínuos (tracejados) correspondem a valores positivos (negativos). Os contornos utilizados são 5, 15, 35, 65. Sombreados escuros (claros) correspondem a valores superiores (inferiores) a 5($5)$. 
Uma comparação direta entre as Figuras 11, 12 e 13 revela uma queda no índice de impacto da previsão de 24 para 48 e 72 horas, particularmente, sobre as regiões continentais. No entanto, embora com um decréscimo geral do índice de impacto da previsão para 48 e 72 horas, o sinal desse índice para a temperatura, a altura geopotencial e o vento zonal, muda de positivo para negativo somente em pequenas regiões. Para a umidade relativa, é notado um decréscimo mais acentuado no índice de impacto em função do tempo de previsão. Ainda, para regiões oceânicas há um acréscimo no valor do índice de impacto no campo de temperatura, o que justifica o aumento no índice de impacto médio espacial para essa variável em 850 $\mathrm{hPa}$, como mostrado na Figura 10.
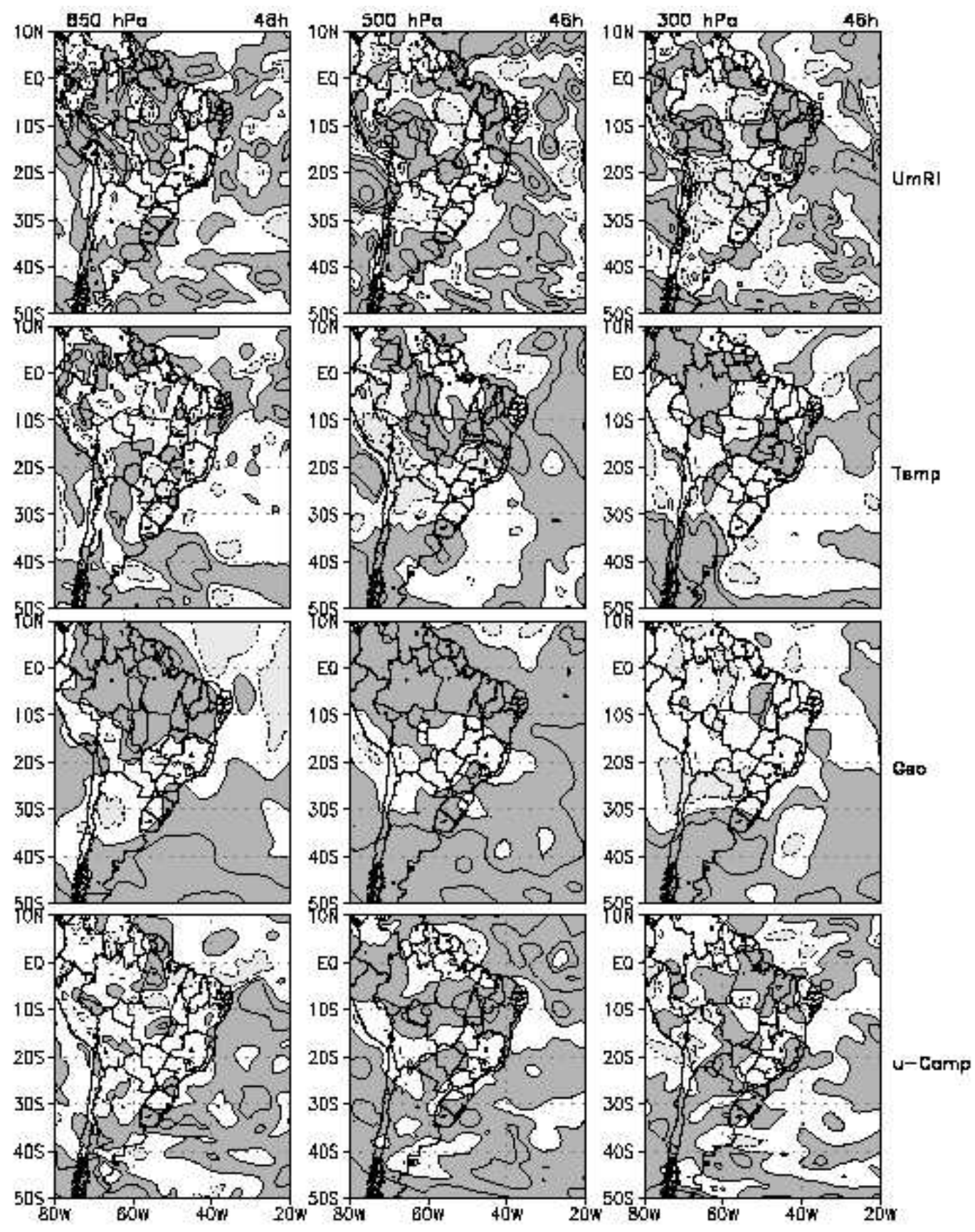

Figura 12 - Idem Figura 11, exceto para 48 horas de integração. 



Figura 13 - Idem Figura 11, exceto para 72 horas de integração.

\section{CONCLUSÕES}

O impacto das sondagens AIRS/AMSU no sistema de assimilação/previsão de tempo do CPTEC é avaliado para o mês de março de 2004. Estatísticas relacionadas às análises de sensibilidade e impacto são apresentadas para 5 variáveis meteorológicos e 5 níveis de pressão. As variáveis consideradas são: temperatura, umidade relativa, umidade específica, altura geopotencial e componente zonal do vento. Análises de sensibilidade nas condições iniciais são calculadas em termos da diferença entre os campos de análise dos experimentos com e sem a inclusão das sondagens AIRS/AMSU e as análises do NCEP. Avaliações de impacto nas previsões são calculadas em termos da diferença entre as previsões e o campo de análise que inclui todas as observações (experimento C_AIRS) e entre previsões e observações (radiossondas). 
Os resultados para os campos de análise e previsões apontam para uma melhora na qualidade dos mesmos, quando as sondagens AIRS/AMSU são incluídas no processo de assimilação de dados, principalmente, em regiões onde o número de observações convencionais é baixo. Além disso, observamse melhorias significativas na performance das previsões sobre o Hemisfério Sul e América do Sul, comparado com os experimentos sem a inclusão dos dados AIRS/AMSU. A melhora na performance da previsão em 4 dias para o Hemisfério Sul é equivalente a ganhar uma extensão da capacidade de previsão em torno de 6 horas. Este resultado indica o considerável potencial das sondagens AIRS/AMSU para melhorar a qualidade das previsões de tempo geradas no CPTEC.

Experimentos considerando a contribuição individual dos diversos sistemas de observação (convencionais ou nãoconvencionais) estão sendo analisados. Além disso, outros experimentos estão sendo realizados com o intuito de avaliar se existe uma dependência temporal (mensal ou sazonal) na performance dos experimentos. Estudos dessa natureza possibilitam um melhor entendimento de como utilizar as informações disponíveis de forma mais eficiente, as quais são essenciais para o aprimoramento da qualidade das análises e das previsões numéricas de tempo geradas diariamente no CPTEC.

\section{AGRADECIMENTOS}

Os autores agradecem o Conselho Nacional de Desenvolvimento Científico e Tecnológico (CNPq), pelo apoio financeiro à pesquisa (Processo 476129/2006-0).

\section{REFERÊNCIAS BIBLIOGRÁFICAS}

ATLAS, R. Impact of AIRS data on numerical weather forecasts at NASA/GSFC. In: Ninth Symposium on Integrated Observing and Assimilation Systems for the Atmosphere, Oceans, and Land Surface (IOAS-AOLS), San Diego, CA. American Meteorological Society, 2005. Session 1: The atmospheric infrared sounder (AIRS).

AUMANN, H. H.; CHAHINE, M. T.; GAUTIER, C.; GOLDBERG, M. D.; KALNAY, E.; MCMILLIN, L. M.; REVERCOMB, H.; ROSENKRANZ, P. W.; SMITH, W. L.; STAELIN, D. H.; STROW, L. L.; SUSSKIND, J. AIRS/AMSU/HSB on the Aqua mission: design, science objectives, data products and processing systems. IEEE Trans. Geosci. Remote Sens., v.41, n.2, p. 253-264, 2003

CAVALCANTI, I. F. A; MARENGO, J. A.; SATYAMURTY, P.; NOBRE, C.; TROSNIKOV, I.; BONATTI, J. P.; MANZI, A. O., TARASOVA, T.; PEZZI, L. P.; D'ALMEIDA, C.; SAMPAIO, G.; CASTRO, C.; SANCHES, M. B.;
CAMARGO, H. Global climatological features in a simulation using the CPTEC-COLA AGCM. J. Climate, v.15, n.21, p. 2965-2988, 2002.

COHN, S. E., DA SILVA, A.; GUO, J.; SIENKIEWICZ, M.; LAMICH, D. Assessing the effects of the data selection with the DAO physical-space statistical analysis system. Mon. Wea. Rev., v.126, p. 2913-2926, 1998.

DA SILVA, A.; PFAENDTNER, J, GUO, J.; SIENKIEWICZ, M.; COHN, S. E. Assessing the effects of Data Selection with the DAO's Physical-space Statistical Analysis System, 1995. Proceedings of the second international symposium on the assimilation of observations in meteorology and oceanography, Tokyo, Japan, WMO and JMA.

DIVAKARLA, M. G.; BARNET, C. D.; GOLDBERG, M. D.; MCMILLIN, L. M.; MADDY, E.; WOLF, W.; ZHOU, L.; LIU, X. Validation of atmospheric Infrared Sounder temperature and water vapor retrievals with matched radiosonde measurements and forecasts. J. Geophys. Res., v. 111, D09S15, doi:10.1029/2005JD006116, 2006.

ERRICO, R. M.; OHRING, G.; DERBER, J; JOINER, J. NOAA-NASA-DoD Workshop on Satellite Data Assimilation. Bull. Amer. Meteor. Soc., v. 81, n. 10, p. 2457-2462, 2000.

EYRE, J. R.; KELLY, A. P.; MCNALLY, A. P.; ANDERSON, E.; PERSON, A. Assimilation of TOVS radiance information trough one-dimensional Variational analysis Quart. J. Roy. Meteor. Soc, v.119, n.514, p. 1427-1463, 1993.

FETZER, E. J. Validation of AIRS/AMSU/HSB core products for Data Release Version 4.0. JPL D-31448, 2005.

HERDIES, D. L.; FERREIRA. S. H.; BONATTI, J. P.; CINTRA, R.; DA SILVA, A. O sistema de assimilação de dados atmosféricos global do CPTEC/INPE. [CD ROM]. In: XII Congresso Brasileiro de Meteorologia, Foz do Iguaçu - PR, 2002. Anais.

JOINER, J.; DA SILVA, A. M. Efficient methods to assimilate Satellite remotely sensed data based on Information content. Quart. J. Roy. Meteor. Soc., v. 124, n. 549, p. 1669-1694, 1998.

KELLY, G., MCNALLY, T.; THEPAUT, J.; SZYNDEL, M. OSE of all main data types in the ECMWF operational system. In: Third WMO Workshop on the Impact of various observing Systems on Numerical Weather Prediction, WMO, Austria, 9-12, March, 2004, Proceedings.

OHRING, G. Impact of Satellite temperature sounding data on weather forecasts. Bull. Amer. Meteor. Soc., v.60, n.10, p. 1142-1147, 1979.

PARKINSON, C. L. Aqua: an Earth-Observing satellite mission to examine water and other climate variables. IEEE Trans. Geosci. Remote Sens., v.41, n.2, p. 173-183, 2003.

SOUZA, R. A. F.; ANDREOLI, R. V.; RODRIGUES, J. 
V.; CEBAllos, J. C.; HERDIES, D.L. Validação das sondagens AQUA sobre a América do Sul. In: XIV Congresso Brasileiro de Meteorologia, 2006, Florianópolis,. Anais. 1 CD-ROM.

SUSSKIND, J.; BARNET, C.; BLAISDELL, J. Retrieval of atmospheric and surface parameters from AIRS/AMSU/ HSB data in the presence of clouds. IEEE Trans. Geosci.
Remote Sens., v.41, n.2, p. 390-409, 2003.

ZAPOTOCNY, T. H., MENZEL, W. P.; JUNG, J. A.; NELSON III, J. P. A Four-Season impact study of rawinsonde, GOES, and POES data in the ETA Data Assimilation system. Part II: Contribution of the Components. Wea. Forecasting, v. 20, n. 2, p. 178-198, 2005. 